\title{
Relationship between the modified embedded-atom method and Stillinger-Weber potentials in calculating the structure of silicon
}

\author{
B. J. Thijsse \\ Laboratory of Materials Science, Delft University of Technology, Rotterdamseweg 137, 2628 AL Delft, Netherlands
}

(Received 9 October 2001; published 7 May 2002)

\begin{abstract}
We show that the Stillinger-Weber (SW) potential is a special case of the modified embedded-atom method (MEAM) potential, by deriving the appropriate functional forms and parameter values for the MEAM potential. The electron density and pair potential functions have physically plausible forms. The embedding function is quadratic in the electron density and yields an antibonding contribution under all circumstances. Using these results SW silicon can be conveniently extended to silicon-metal systems within one theoretical framework and one computational scheme. The properties of SW silicon and silicon modeled by the native MEAM representation are compared.
\end{abstract}

DOI: 10.1103/PhysRevB.65.195207

PACS number(s): 61.43.Bn, 61.50.Ah, 83.10.Rs

\section{INTRODUCTION}

Computational modeling of materials at an atomic level is increasingly concerned with large and complex systems, often containing different atomic species. In spite of the current progress of $a b$ initio calculations, classical simulations using semiempirical potentials are still needed when systems of more than a few hundred atoms are studied. Traditionally the development of potentials for metals and semiconductors has taken place separately and in different formats, which is not surprising because of the difference in their bonding nature. Many metals can be well described by potentials that do not take into account angle-dependent terms, whereas for semiconductors this is impossible. Over the last decade the embedded-atom method ${ }^{1}$ (EAM) has been among the most popular modeling schemes for metals, while the Tersoff ${ }^{2}$ and especially the Stillinger-Weber ${ }^{3}$ (SW) potentials are the most commonly used for silicon.

The question can be raised, however, whether these potential classes are truly different. Already in 1989 Brenner showed that the Tersoff potential is identical to the EAM potential for a reasonable choice of functions and parameters. ${ }^{4}$ However, the angular dependence of the Tersoff potential had to be omitted in this comparison, which limits the scope of the discovered identity considerably. Metals and semiconductors still could not be combined in one computational scheme. Shortly thereafter Baskes developed the full version of the modified embedded-atom method ${ }^{5}$ (MEAM), which adds angular terms having $s, p, d$, and $f$ symmetries to the EAM. Not only is the MEAM computationally efficient, its framework is also sufficiently flexible to handle simple metals, transition metals with their weak yet significant angular interactions, and semiconductors. Several authors have exploited these possibilities; examples can be found in Refs. $6-10$.

In this paper we show that the introduction of the MEAM potential has by no means left the SW potential in an isolated position. Without invoking approximations we show that the SW potential is not different from the MEAM potential, but rather a special case of it. More specifically, we derive the forms of the MEAM functions and the values of the MEAM parameters that cause the MEAM potential to behave exactly like the SW potential. This makes it possible to combine "Stillinger-Weber silicon" with metallic elements in atomistic simulations, from which various types of research-for example, that on silicides, Al-Si alloys, and some metallic glasses-will benefit greatly. As mentioned above, silicon also has a "native" representation in terms of the MEAM formalism, which allows an integrated study of metal-silicon systems as well. However, there already exists a large body of data on silicon modeled with the SW potential; to be able to extend these to silicon combined with metals would be very useful, even more so because the native MEAM representation of silicon is less extensively tested. Casting the SW potential into the MEAM format also has theoretical implications, since it will become clear how the SW interaction can be interpreted in terms of the MEAM concepts such as local electron density and embedding energy. Moreover, the additional freedom of the MEAM formalism offers logical ways to refine the SW potential within a well-defined theoretical framework. This may be useful, since various shortcomings of the SW potential have been identified; see, e.g., Ref. 11. Conversely, the parameters of the native MEAM representation may be brought closer to those of the SW version in order to change those properties of silicon that are predicted better by the SW model than by the native model; a comparison of some important silicon properties is given at the end of this paper. Finally, the present results allow users of the MEAM potentials to switch easily to SW silicon or to study more complex systems that are formulated in terms of the SW potential, such as the silicon-oxygen system, where charge transfer should be taken into account. ${ }^{12}$ Recently Lenosky et al. have published a quite elaborate MEAM-like potential for silicon. ${ }^{13}$ In their work they briefly mention that the SW potential is a special case of their potential model, ${ }^{14}$ but they do not pursue the issues addressed here. Carlsson ${ }^{15}$ has shown that the SW potential is a special case of the Biswas-Hamman potential. ${ }^{16}$

\section{THEORY}

\section{A. Stillinger-Weber potential}

The SW potential expresses the energy as a sum of pair and triplet contributions, ${ }^{3}$ 
TABLE I. Parameters of the Stillinger-Weber potential (Ref. 3) and the native MEAM representation of Si (Ref. 17). The original values of $A$ and $\lambda$ have been multiplied by 1.0676 so that the cohesive energy of Si becomes $4.63 \mathrm{eV}$ instead of $4.34 \mathrm{eV}$ (Ref. 11). The fixed length $\sigma$ is included for convenience.

\begin{tabular}{lccccc}
\hline \hline \multicolumn{2}{c}{ Stillinger-Weber } & \multicolumn{4}{c}{ Native MEAM } \\
\hline$A(\mathrm{eV})$ & 16.319 & $t^{(1)}$ & 2.05 & $\beta^{(3)}$ & 5.5 \\
$B$ & 0.60222 & $t^{(2)}$ & 4.47 & $r_{e}(\AA)$ & 2.350 \\
$p$ & 4 & $t^{(3)}$ & -1.8 & $A_{E}$ & 1.00 \\
$q$ & 0 & $f_{e}$ & 0.1705 & $E_{c}(\mathrm{eV})$ & 4.63 \\
$a(\AA)$ & 3.7712 & $\beta^{(0)}$ & 4.4 & $\alpha$ & 4.893 \\
$\lambda(\mathrm{eV})$ & 48.615 & $\beta^{(1)}$ & 5.5 & $r_{s}(\AA)$ & 2.945 \\
$\gamma(\AA)$ & 2.5141 & $\beta^{(2)}$ & 5.5 & $r_{c}(\AA)$ & 3.540 \\
$\sigma(\AA)$ & 2.0951 & & & & \\
\hline \hline
\end{tabular}

$$
E=\sum_{i} \sum_{j<i} v_{2}\left(r_{i j}\right)+\sum_{i} \sum_{j<i} \sum_{k<j} v_{3}(i, j, k)
$$

with

$$
\begin{gathered}
v_{2}\left(r_{i j}\right)=A\left(B\left(r_{i j} / \sigma\right)^{-p}-\left(r_{i j} / \sigma\right)^{-q}\right) e^{\left(r_{i j} / \sigma-a\right)^{-1}}, \\
v_{3}(i, j, k)=h_{j i k}+h_{i j k}+h_{i k j}, \\
h_{j i k}=\lambda e^{\gamma\left(r_{i j} / \sigma-a\right)^{-1}+\gamma\left(r_{i k} / \sigma-a\right)^{-1}}\left(\cos \theta_{j i k}+\frac{1}{3}\right)^{2} .
\end{gathered}
$$

In these equations $r_{i j}$ is the distance between atoms $i$ and $j$, and it is implied that $r_{i j}$ and $r_{i k}$ are smaller than $a \sigma$. If not, the corresponding term $v_{2}\left(r_{i j}\right)$ or $h_{j i k}$ vanishes. The angle $\theta_{j i k}$ is the triplet angle subtended at atom $i$. The quantity $\sigma$ sets the length scale and has a fixed value of $2.0951 \AA$. The values of the seven parameters $A, B, p, q, a, \lambda, \gamma$ were given by Stillinger and Weber ${ }^{3}$ and are listed in Table I.

\section{B. Modified embedded-atom method potential}

The MEAM potential expresses the energy as a sum of pair and (environment-dependent) single-atom contributions, ${ }^{5,17}$

$$
E=\sum_{i} \sum_{j \neq i} \frac{1}{2} \phi\left(r_{i j}\right)+\sum_{i} F\left(\rho_{i}\right),
$$

in which $\phi$ is the pair potential, $F$ the embedding energy function, and $\rho_{i}$ the electron density at the position of atom $i$. This electron density is a function of the electron densities contributed by the atoms surrounding $i$. In the original work on MEAM, ${ }^{5} \rho_{i}$ was expressed as

$$
\rho_{i}=\left(\sum_{l=0}^{3} t^{(l)}\left(\rho_{i}^{(l)}\right)^{2}\right)^{1 / 2}
$$

in which the parameters $t^{(l)}, l=1-3$, are weight factors $\left(t^{(0)}=1\right)$ and the quantities $\rho_{i}^{(l)}$ are partial electron densities. In later work, ${ }^{18} \rho_{i}$ is expressed as

$$
\rho_{i}=\frac{2 \rho_{i}^{(0)}}{1+\exp \left[-\sum_{l=1}^{3} t^{(l)}\left(\rho_{i}^{(l)} / \rho_{i}^{(0)}\right)^{2}\right]},
$$

which avoids the possibility of an imaginary $\rho_{i}$ in case of negative $t^{(l)}$ values. The partial electron densities are given by ${ }^{19}$

$$
\left(\rho_{i}^{(l)}\right)^{2}=\sum_{j \neq i} \sum_{k \neq i} f^{(l)}\left(r_{i j}\right) f^{(l)}\left(r_{i k}\right) L^{(l)}\left(\cos \theta_{j i k}\right),
$$

where the functions $L^{(l)}, l=0-3$, are the (unnormalized) Legendre polynomials, $L^{(0)}(z)=1, L^{(1)}(z)=z, L^{(2)}(z)=z^{2}$ $-\frac{1}{3}$, and $L^{(3)}(z)=z^{3}-\frac{3}{5} z$. The form of Eq. (8) differs from the one given in Ref. 5 , because it is more convenient for the present analysis; mathematically, they are equivalent. The functions $f^{(l)}, l=0-3$, express the partial electron density contributions from the atoms surrounding $i$,

$$
f^{(l)}\left(r_{i j}\right)=f_{e} e^{-\beta^{(l)}\left(r_{i j} / r_{e}-1\right)},
$$

where $r_{e}$ is the equilibrium nearest-neighbor distance in the perfect crystal and $f_{e}$ and the numbers $\beta^{(l)}, l=0-3$, are parameters. In this work we have used a smooth cubic cutoff polynomial between $r_{s}=2.945 \AA$ and $r_{c}=3.540 \AA$ to limit the electron range. The embedding function is

$$
F\left(\rho_{i}\right)=A_{E} E_{c}\left(\frac{\rho_{i}}{\rho_{X}\left(r_{e}\right)}\right) \ln \left(\frac{\rho_{i}}{\rho_{X}\left(r_{e}\right)}\right),
$$

with $A_{E}$ a parameter, $E_{c}$ the cohesion energy of the equilibrium crystal, and $\rho_{X}\left(r_{e}\right)$ the electron density at the site of an atom in the equilibrium crystal. This form of $F$ shows that in monatomic systems the value of $f_{e}$ is irrelevant, since it cancels out of the density ratio. Here we will use $f_{e}=0.1705$ for reasons explained below. The pair potential $\phi$ is constructed in such a way that the energy of an atom in the perfect crystal varies with the nearest-neighbor distance $R$ exactly as the universal relation proposed by Rose et al. ${ }^{20}$ When only nearest-neighbor interactions are considered, as is the case for silicon, the pair potential then becomes

$$
\begin{aligned}
\phi(R)= & -2 \frac{E_{c}}{Z}\left[\left[1+\eta+0.05 \eta^{3}\right] e^{-\eta}\right. \\
& \left.+A_{E}\left(\frac{\rho_{X}(R)}{\rho_{X}\left(r_{e}\right)}\right) \ln \left(\frac{\rho_{X}(R)}{\rho_{X}\left(r_{e}\right)}\right)\right],
\end{aligned}
$$

with $Z$ the coordination number and $\eta$ a parameter given by

$$
\eta=\alpha\left(\frac{R}{r_{e}}-1\right),
$$

where $\alpha=\left(9 \Omega_{e} B / E_{c}\right)^{1 / 2}$, with $\Omega_{e}$ the equilibrium atomic volume and $B$ the bulk modulus. We will refer to Eqs. (9)(12) as the "native" MEAM representation. The 14 parameter values for Si (Ref. 17) are given in Table I. 


\section{Relation between the two potentials}

In order to show that the SW energy of Eq. (1) can be written in the form of Eq. (5), we start by separating the pair terms $(j=k)$ and triplet terms $(j \neq k)$ of Eq. (8),

$$
\begin{aligned}
\left(\rho_{i}^{(l)}\right)^{2}= & 2 \sum_{j, k \subset T_{i}} f^{(l)}\left(r_{i j}\right) f^{(l)}\left(r_{i k}\right) L^{(l)}\left(\cos \theta_{j i k}\right) \\
& +\sum_{j \neq i}\left[f^{(l)}\left(r_{i j}\right)\right]^{2} L^{(l)}(1)
\end{aligned}
$$

in which the notation $j, k \subset T_{i}$ refers to all atom pairs $j, k$ that form unique triplets with atom $i$. Combining this with Eq. (6) leads to

$$
\rho_{i}^{2}=\left(\rho_{i}^{T}\right)^{2}+\left(\rho_{i}^{P}\right)^{2},
$$

where the triplet and pair contributions are given by

$$
\begin{gathered}
\left(\rho_{i}^{T}\right)^{2}=\sum_{j, k \subset T_{i}}\left\{\sum_{l=0}^{3} 2 t^{(l)} f^{(l)}\left(r_{i j}\right) f^{(l)}\left(r_{i k}\right) L^{(l)}\left(\cos \theta_{j i k}\right)\right\}, \\
\left(\rho_{i}^{P}\right)^{2}=\sum_{j \neq i}\left\{\sum_{l=0}^{3} t^{(l)}\left[f^{(l)}\left(r_{i j}\right)\right]^{2} L^{(l)}(1)\right\} .
\end{gathered}
$$

When we next take $f^{(l)}\left(r_{i j}\right)$ as

$$
f^{(l)}\left(r_{i j}\right)=e^{\gamma\left(r_{i j} / \sigma-a\right)^{-1}}
$$

for all $l$, and we choose the parameters $t^{(l)}$ as

$$
t^{(0)}=1, \quad t^{(1)}=\frac{3}{2}, \quad t^{(2)}=\frac{9}{4}, \quad t^{(3)}=0,
$$

Eq. (15) becomes

$$
\left(\rho_{i}^{T}\right)^{2}=\frac{9}{2 \lambda} \sum_{j, k \subset T_{i}} h_{j i k} .
$$

In the triple summation in Eq. (1) only unique triplets are counted; however, each triplet contributes three energy terms, each of which can be attributed to a different atom of the triplet: $h_{j i k}$ to atom $i, h_{i j k}$ to atom $j$, and $h_{i k j}$ to atom $k$. Therefore,

$$
\sum_{i} \sum_{j<i} \sum_{k<j} v_{3}(i, j, k)=\frac{2}{9} \lambda \sum_{i}\left(\rho_{i}^{T}\right)^{2} .
$$

Furthermore, evaluation of Eq. (16), using Eq. (18) yields

$$
\left(\rho_{i}^{P}\right)^{2}=4 \sum_{j \neq i}\left[f\left(r_{i j}\right)\right]^{2},
$$

where we have dropped the superscript $(l)$ from $f$. With these results we can finally write Eq. (1) as

$$
E=\sum_{i} \sum_{j \neq i} \frac{1}{2}\left[v_{2}\left(r_{i j}\right)-\frac{16}{9} \lambda\left[f\left(r_{i j}\right)\right]^{2}\right]+\sum_{i} \frac{2}{9} \lambda \rho_{i}^{2} .
$$

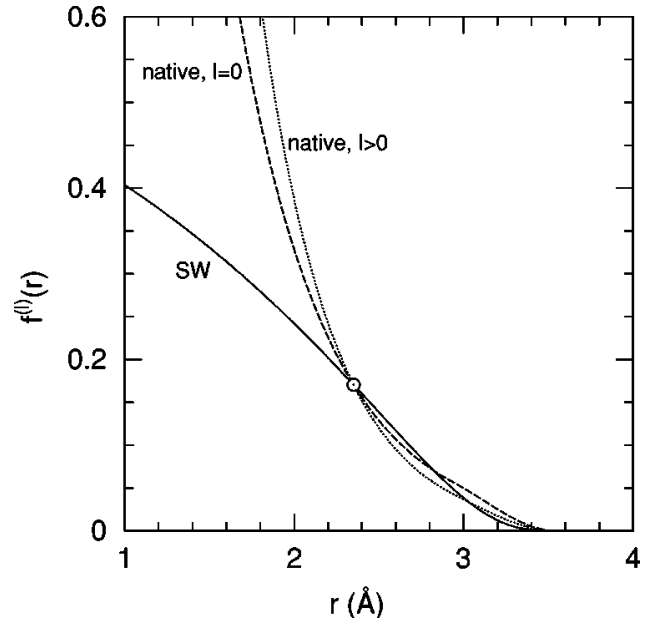

FIG. 1. Electronic density functions $f^{(l)}(r)$ for the SW-MEAM and native MEAM models. The circle marks the conditions in the equilibrium silicon crystal.

This shows that the MEAM potential is identical to the SW potential if the embedding function is taken as

$$
F\left(\rho_{i}\right)=\frac{2}{9} \lambda \rho_{i}^{2}
$$

and the pair potential as

$$
\phi\left(r_{i j}\right)=v_{2}\left(r_{i j}\right)-\frac{16}{9} \lambda\left[f\left(r_{i j}\right)\right]^{2} .
$$

The electron densities $\rho_{i}$ at the atomic positions should be calculated according to Eq. (6), using the parameter values of Eq. (18), and $f\left(r_{i j}\right)$ should be computed according to Eq. (17). These are the main results of this paper.

\section{DISCUSSION}

The two different models of silicon-SW-MEAM and native MEAM-can now be compared in terms of electron density, embedding energy, and pair potential. The native MEAM model was evaluated using Eq. (7), since for silicon Eq. (6) yields imaginary electron densities in the calculation of the pair potential at small interatomic separations.

Figure 1 shows the electron density functions $f^{(l)}(r)$. The value $f_{e}=0.1705$ was chosen so that the electron densities of the SW and native models have the same value at the equilibrium Si-Si distance $r_{e}$, which facilitates comparison. Note that the density is expressed as a dimensionless number. It is seen that above $r_{e}$ the two models behave similarly and the electron densities vanish at about the same distance. At distances smaller than $r_{e}$ the native model displays a fast increasing electron density while the SW model exhibits a slower increase. This difference will be felt by other elements if they are part of the system and should be accommodated by a mixed pair potential different from the native MEAM version.

Figure 2 shows the two embedding functions. In the native model the embedding energy in the equilibrium crystal is zero, which means that the cohesion is entirely due to the 


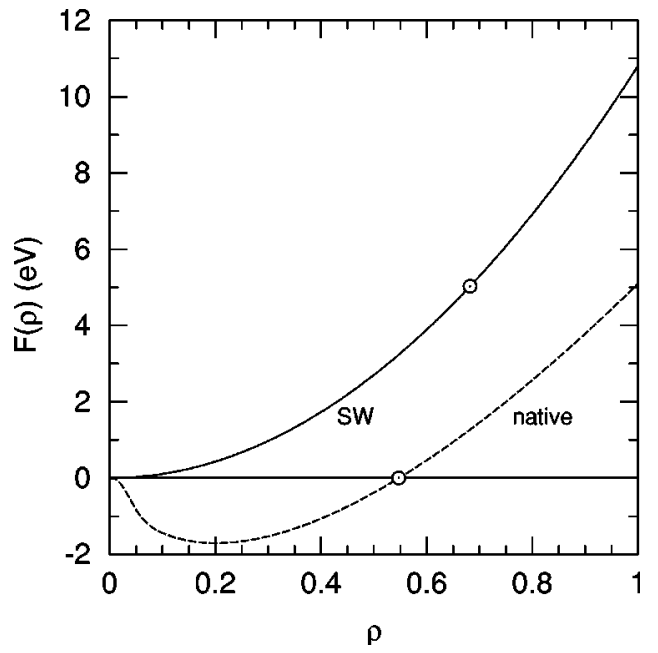

FIG. 2. Embedding functions $F(\rho)$ for the SW-MEAM and native MEAM models. Close to $\rho=0$ the native embedding function has been smoothed to remove the singularity in its derivative. The circles mark the conditions in the equilibrium silicon crystal.

pair potential. This is somewhat at variance with the physical picture of embedding, which was originally regarded as a bonding mechanism. In the SW model the embedding energy in the equilibrium crystal is even antibonding $(+5.02 \mathrm{eV})$. In fact, it is antibonding for every electron density. This point is not as significant as it seems. It is well known that in the EAM one can arbitrarily reestablish the bonding character of the embedding function by subtracting an appropriate term linear in the electron density. This is because adding a corresponding term to the pair potential leaves the total energy unchanged. ${ }^{21}$ Although in the MEAM this is formally not possible, since the electron density no longer has the form of a pair potential, a similar reformulation of $F$ followed by a new parameter fit is nevertheless equally likely to succeed. Perhaps it is best to take the idea of embedding not too literally. This conclusion agrees with van Beurden and Kramer, ${ }^{22}$ who argue that $\rho_{i}$ should be interpreted as the effective coordination number of atom $i$, rather than as the local electron density, and $F$ as a bond order correction function. Mishin $e t$ al. $^{23}$ practically abandon all physical interpretation and consider the functions as mere fitting functions. Apart from this matter concerning the sign of $F$ the two embedding curves have similar shapes, and have derivatives of comparable magnitudes.

The pair potentials are displayed in Fig. 3. The oscillation in the native pair potential above $r=3 \AA$ is the result of the implicit requirement that the universal relation of Rose et al. should be obeyed for a silicon crystal that is expanded without limits. Here this requirement is dropped for large expansions, and the pair potential is smoothly brought to zero between $r_{s}=2.945 \AA$ and $r_{c}=3.540 \AA$, in accordance with the electron density functions. When this is done, the two pair potentials look very much the same. The SW pair potential is deeper by a factor $\approx 1.9$; this is because it has to compensate for the antibonding contribution of the embedding energy. Interestingly, the two curves have their minimum at very nearly the same value of $r(1.94 \AA)$. This is

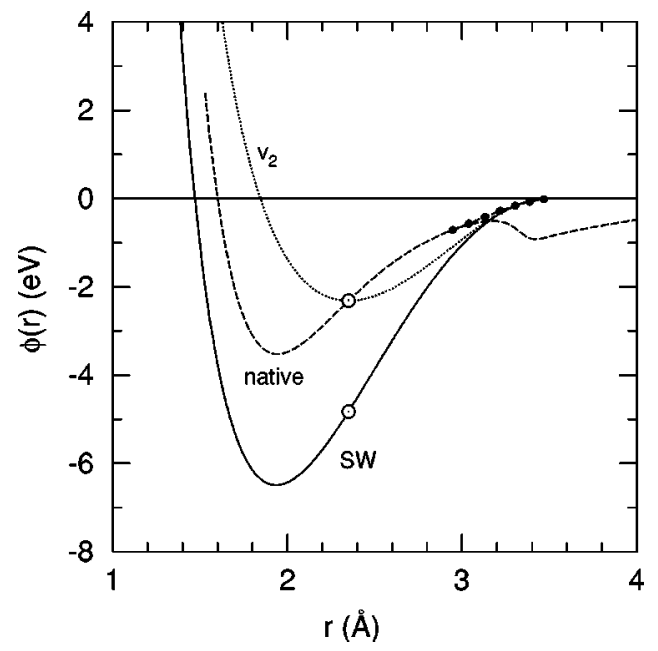

FIG. 3. Pair potentials $\phi(r)$ for the SW-MEAM and native MEAM models. The black dots indicate the shorter-range version of the native pair potential, which is used in practice. The curve labeled $v_{2}$ is the original SW pair potential. The circles mark the conditions in the equilibrium silicon crystal.

entirely due to the term $-\frac{16}{9} \lambda\left[f\left(r_{i j}\right)\right]^{2}$ in the SW-MEAM pair potential; the original SW pair potential $v_{2}\left(r_{i j}\right)$ is quite different and has its minimum in the higher of the two circles shown in Fig. 3.

TABLE II. Selected properties of silicon: elastic constants $C_{11}$, $C_{12}, C_{44}$, thermal expansion coefficient $\alpha_{\mathrm{T}}$ (at $300 \mathrm{~K}$ ), unrelaxed and relaxed vacancy formation energies $E_{u}^{v}$ and $E_{r}^{v}$, dimer cohesion energy $E_{2}$ and bond length $r_{2}$, equilateral trimer cohesion energy $E_{3}$ and bond length $r_{3}$, surface energy $\gamma_{(100) 2 \times 1}$ of the $2 \times 1$ reconstructed (100) surface, and sputter yield $Y_{500}$ during prolonged bombardment of a $\mathrm{Si}(100)$ surface by a $500 \mathrm{eV} \mathrm{Ar}^{+}$beam impinging at $45^{\circ}$. Unless indicated otherwise, Stillinger-Weber and native MEAM results are from this work. Results from quantum calculations of various types (QC) are indicated by an asterisk.

\begin{tabular}{lccc}
\hline \hline Property & $\begin{array}{c}\text { Stillinger- } \\
\text { Weber }\end{array}$ & $\begin{array}{c}\text { Native } \\
\text { MEAM }\end{array}$ & $\begin{array}{c}\text { Experimental, } \\
\text { QC* }\end{array}$ \\
\hline$C_{11}(\mathrm{GPa})$ & 162 & 178 & $166^{\mathrm{a}}$ \\
$C_{12}(\mathrm{GPa})$ & 82 & 57 & $64^{\mathrm{a}}$ \\
$C_{44}(\mathrm{GPa})$ & 60 & 93 & $80^{\mathrm{a}}$ \\
$\alpha_{\mathrm{T}}\left(10^{-6} \mathrm{~K}^{-1}\right)$ & 4.3 & 12.6 & $2.6^{\mathrm{b}}$ \\
$E_{u}^{v}(\mathrm{eV})$ & 4.63 & 4.47 & $4.12^{* \mathrm{c}}$ \\
$E_{r}^{v}(\mathrm{eV})$ & 2.82 & 4.16 & $3.73^{* \mathrm{c}}$ \\
$E_{2}(\mathrm{eV})$ & 2.32 & 5.15 & $3.24^{\mathrm{a}}$ \\
$r_{2}(\AA)$ & 2.35 & 2.42 & $2.25^{\mathrm{a}}$ \\
$E_{3}(\mathrm{eV})$ & 4.74 & 8.00 & $7.6^{* \mathrm{a}}$ \\
$r_{3}(\AA)$ & 2.56 & 2.68 & $2.26^{* \mathrm{a}}$ \\
$\gamma_{(100) 2 \times 1}\left(\mathrm{Jm}^{-2}\right)$ & 1.54 & 1.38 & $1.7^{* \mathrm{a}}$ \\
$Y_{500}\left(\mathrm{Si} / \mathrm{Ar}^{+}\right)$ & $1.7^{\mathrm{d}}$ & $3.5^{\mathrm{d}}$ & $1.7^{\mathrm{e}}$ \\
\hline \hline
\end{tabular}

${ }^{\mathrm{a}}$ Reference 11 .

${ }^{\mathrm{b}}$ Reference 24 .

${ }^{\mathrm{c}}$ Reference 13 .

${ }^{\mathrm{d}}$ Reference 25 .

${ }^{\mathrm{e}}$ Reference 26. 
All of this is reassuring. The MEAM representation of the SW potential leads to physically plausible forms of the MEAM functions. This indicates that the MEAM offers a natural framework in which SW silicon can be combined with metallic elements. Nevertheless, the differences between the SW and native MEAM representations themselves are too large to step over lightly. They suggest that the properties of silicon may well be quite different in the two models. Table II, which collects property values calculated with the two potentials and compares them with experimental values (and results from quantum calculations), shows that this is indeed the case. Both the SW and the native MEAM models have their shortcomings. Going over Table II from top to bottom, from conditions close to the equilibrium crystal to conditions far from equilibrium, one notices that the SW model is particularly poor in predicting the sign of $C_{12}$ $-C_{44}$ (the elastic Cauchy discrepancy) and the values of the relaxed vacancy formation energy and the dimer and trimer cohesion energies, and that the native MEAM notably fails in predicting the thermal expansion coefficient, the dimer en- ergy, the trimer bond length, and the sputter yield. This is a situation that clearly calls for improvement. It is not the intention of this paper to indicate how the SW and native MEAM parameters should be modified to remove the shortcomings in their predictions; this has to be postponed to a future paper. Rather, with the results in this work we have shown that such modifications can be conveniently carried out in one common theoretical framework.

\section{ACKNOWLEDGMENTS}

We thank P. van Beurden, T.P.C. Klaver, and E.F.C. Haddeman for their careful reading of the manuscript. This work is part of the research program of the Stichting voor Fundamenteel Onderzoek der Materie (Foundation for Fundamental Research on Matter) and was made possible by financial support from the Nederlandse Organisatie voor Wetenschappelijk Onderzoek NWO (Netherlands Organization for Scientific Research).
${ }^{1}$ M.S. Daw and M.I. Baskes, Phys. Rev. B 29, 6443 (1984).

${ }^{2}$ J. Tersoff, Phys. Rev. B 37, 6991 (1988); 38, 9902 (1988).

${ }^{3}$ F.H. Stillinger and T.A. Weber, Phys. Rev. B 31, 5262 (1985).

${ }^{4}$ D.W. Brenner, Phys. Rev. Lett. 63, 1022 (1989).

${ }^{5}$ M.I. Baskes, Phys. Rev. B 46, 2727 (1992).

${ }^{6}$ T.E. Mitchell, M.I. Baskes, S.P. Chen, J.P. Hirth, and R.G. Hoagland, Philos. Mag. A 81, 1079 (2001).

${ }^{7}$ J.F. Aguilar, R. Ravelo, and M.I. Baskes, Modell. Simul. Mater. Sci. Eng. 8, 335 (2000).

${ }^{8}$ K. Takahashi, C. Nara, T. Yamagishi, and T. Onzawa, Appl. Surf. Sci. 151, 299 (1999).

${ }^{9}$ D. Chen, M. Yan, and Y.F. Liu, Scr. Mater. 40, 913 (1999).

${ }^{10}$ Zhang Bangwei, Ouyang Yifang, Liao Shuzhi, and Jin Zhanpeng, Physica B 262, 218 (1999).

${ }^{11}$ H. Balamane, T. Halicioglu, and W.A. Tiller, Phys. Rev. B 46, 2250 (1992).

${ }^{12}$ Z. Jiang and R.A. Brown, Phys. Rev. Lett. 74, 2046 (1995).

${ }^{13}$ T.J. Lenosky, B. Sadigh, E. Alonso, V.V. Bulatov, T. Diaz de la Rubia, J. Kim, A.F. Voter, and J.D. Kress, Modell. Simul. Mater. Sci. Eng. 8, 825 (2000).

${ }^{14}$ In Ref. 13, $\rho=0$ was incorrectly mentioned as one of the conditions.
${ }^{15}$ A.E. Carlsson, Solid State Phys. 43, 1 (1990).

${ }^{16}$ R. Biswas and D.R. Hamann, Phys. Rev. B 36, 6434 (1987).

${ }^{17}$ M.I. Baskes, Mater. Sci. Eng., A 261, 165 (1999).

${ }^{18}$ M.I. Baskes, Mater. Chem. Phys. 50, 152 (1997).

${ }^{19}$ R. Biswas and D.R. Hamann, Phys. Rev. Lett. 55, 2001 (1985).

${ }^{20}$ J.H. Rose, J.R. Smith, F. Guinea, and J. Ferrante, Phys. Rev. B 29, 2963 (1984).

${ }^{21}$ M.W. Finnis and J.E. Sinclair, Philos. Mag. A 50, 45 (1984).

${ }^{22}$ P. van Beurden and G.J. Kramer, Phys. Rev. B 63, 165106 (2001).

${ }^{23}$ Y. Mishin, M.J. Mehl, D.A. Papaconstantopoulos, A.F. Voter, and J.D. Kress, Phys. Rev. B 63, 224106 (2001).

${ }^{24}$ Table of Physical and Chemical Constants, 16th ed., originally compiled by G. W. C. Kaye and T. H. Laby (Langman, Harlow, UK, 1995).

${ }^{25}$ E.F.C. Haddeman and B.J. Thijsse (unpublished). The sputter yield was determined from molecular-dynamics calculations in which the Molière-screened Coulomb potential [see, e.g., Atomic and Ion Collisions in Solids and at Surfaces, edited by R. Smith (Cambridge University, Cambridge, England, 1997)] was smoothly connected to the SW and native MEAM potentials to describe the high-energy interactions.

${ }^{26}$ J.G.M. van Berkum (unpublished). 